\title{
Chiral Order and Multiferroic Domain Relaxation in $\mathrm{NaFeGe}_{2} \mathrm{O}_{6}$
}

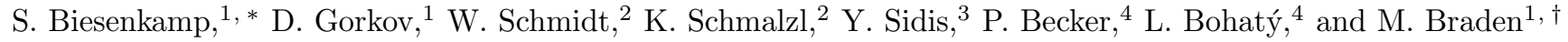 \\ ${ }^{1}$ II. Physikalisches Institut, Universität zu Köln, Zülpicher Straße 77, D-50937 Köln, Germany \\ ${ }^{2}$ Juelich Centre for Neutron Science JCNS, Forschungszentrum Juelich GmbH, Outstation at ILL, 38042 Grenoble, France \\ ${ }^{3}$ UniversitéParis-Saclay, CNRS, CEA, Laboratoire Léon Brillouin, F-91191, Gif-sur-Yvette, France \\ ${ }^{4}$ Abteilung Kristallographie, Institut für Geologie und Mineralogie, \\ Universität zu Köln, Zülpicher Straße 49b, 50674 Köln, Germany
}

(Dated: May 17, 2021)

\begin{abstract}
The magnetic structure and the multiferroic relaxation dynamics of $\mathrm{NaFeGe}_{2} \mathrm{O}_{6}$ were studied by neutron scattering on single crystals partially utilizing polarization analysis. In addition to the previously reported transitions, the incommensurate spiral ordering of $\mathrm{Fe}^{3+}$ moments in the $a c$ plane develops an additional component along the crystallographic $b$ direction below $T \approx 5 \mathrm{~K}$, which coincides with a lock-in of the incommensurate modulation. The quasistatic control of the spin-spiral handedness, respectively of the vector chirality, by external electric fields proves the invertibility of multiferroic domains down to the lowest temperature. Time-resolved measurements of the multiferroic domain inversion in $\mathrm{NaFeGe}_{2} \mathrm{O}_{6}$ reveal a simple temperature and electric-field dependence of the multiferroic relaxation that is well described by a combined Arrhenius-Merz relation, as it has been observed for $\mathrm{TbMnO}_{3}$. The maximum speed of domain wall motion is comparable to the spin wave velocity.
\end{abstract}

\section{INTRODUCTION}

The increasing power consumption due to data storage and the growing amount of information that has to be stored or buffered enforce the development of new and more effectively working memory devices [1, 2]. Beside, phase change based devices, skyrmion racetracks and other promising techniques [2 9], multiferroic systems are promising for designing future storage techniques [10, 11].

In multiferroics at least two ferroic ordering parameters occur in the same phase and are coupled to each other 12. Both ordering parameters can be controlled by both conjugate fields. The magnetoelectric case with ferroelectric and magnetic ordering is most prominent, as it allows for a multiferroic memory, which combines the advantages of ferroelectric (FeRAMs) and magnetic random access memories (MRAMs) 10, 11, 13. In so-called typeII multiferroics the ferroelectric polarization is not only coexisting with magnetic ordering but it is also induced by the onset of it, which hence implies strong coupling between both ferroic ordering parameters [12. In many systems the driving mechanism for this effect is the inverse Dzyaloshinskii-Moriya interaction (DMI), for which a spin canting of neighboring spins $\boldsymbol{S}_{i}$ and $\boldsymbol{S}_{j}$ induces a ferroelectric polarization, whose direction is defined by $\boldsymbol{P} \propto \boldsymbol{e}_{i j} \times\left(\boldsymbol{S}_{i} \times \boldsymbol{S}_{i}\right)$ with $\boldsymbol{e}_{i j}$ being the connecting vector of neighboring spins [14-16].

The understanding of the read and write performance under different conditions is indispensable for the development of new memory devices. The control and the dynamics of multiferroic domain inversion were studied intensively for $\mathrm{TbMnO}_{3}$ and $\mathrm{MnWO}_{4}$ using neutron scattering techniques, second-harmonic generation

\footnotetext{
* e-mail: biesenkamp@ph2.uni-koeln.de

$\dagger$ e-mail: braden@ph2.uni-koeln.de
}

(SHG) measurements and dielectric spectroscopy [17 21 . The relaxation behavior in both systems differs significantly as a function of temperature and electric-field amplitude [18 20]. In $\mathrm{MnWO}_{4}$ domain inversion speeds up, when cooling towards the lower commensurate magnetic phase [18, 19], which is attributed to depinning of multiferroic domains by anharmonic modulations [22, 23. In $\mathrm{TbMnO}_{3}$ the domain inversion can be well described by a remarkably simple law combining an Arrhenius-like activated temperature dependence with a field dependence following the Merz law established for ferroelectrics [20]. In contrast to $\mathrm{MnWO}_{4}$, the incommensurate long-range order in multiferroic $\mathrm{TbMnO}_{3}$ does not transform into a commensurate arrangement 24, 25] but is only altered through the additional order of $\mathrm{Tb}^{3+}$ moments [26. Apparently, competing incommensurate and commensurate ordering significantly influence the relaxation behavior of multiferroic domain inversion and thus complicate its description [23]. Therefore, multiferroic materials with simple phase diagrams are desirable for further investigations of multiferroic domain dynamics.

The broad class of pyroxenes with chemical formula $A M e X_{2} \mathrm{O}_{6}$ with $A=\mathrm{Li}, \mathrm{Na}, \mathrm{Sr}$ or $\mathrm{Ca}, X=\mathrm{Si}$ or $\mathrm{Ge}$ and $M e$ a magnetic transition metal exhibit several interesting magnetic ordering phenomena, with controllable ferrotoroidicity in $\mathrm{LiFeSi}_{2} \mathrm{O}_{6}$ 27, 28, and multiferroic phases in $\mathrm{NaFeSi}_{2} \mathrm{O}_{6}$ 27, in $\mathrm{NaFeGe}_{2} \mathrm{O}_{6} 29$ and in $\mathrm{SrMnGe}_{2} \mathrm{O}_{6}$ [30] being the most prominent ones. In spite of the similar monoclinic crystal structure with magnetic zigzag chains running along the $c$ direction, see Fig. 1, the multiferroic mechanism is different in these three pyroxenes. In $\mathrm{NaFeSi}_{2} \mathrm{O}_{6}$ two successive transitions involving the same irreducible representation result in a helical structure with the propagation vector along the monoclinic axis [31]. In this material multiferroic order does not result from the spin-current or inverse DzyaloshinskiMoriya mechanism 14, 16, 32 but from the combina- 
tion of the chiral order and the monoclinic distortion. In contrast, multiferroic order in $\mathrm{SrMnGe}_{2} \mathrm{O}_{6}$ 30 and in $\mathrm{NaFeGe}_{2} \mathrm{O}_{6}$ [3] seems to follow the most common inverse Dzyaloshinski-Moriya mechanism [14-16, 32 but with differently oriented cycloidal and ferroelectric order.

$\mathrm{NaFeGe}_{2} \mathrm{O}_{6}$ exhibits a simple phase diagram with the typical sequence of two incommensurate ordered phases and with just one magnetic site [29, 33 36]. The system crystallizes in the monoclinic spacegroup $C 2 / c$ with $a=10.0073(8) \AA, b=8.9382(7) \AA, c=5.5184(4) \AA$ and $\beta=107.524(1)^{\circ}$ 35, 37. Edge sharing $\mathrm{FeO}_{6}$ octahedra form zigzag chains along the $c$ direction and in $b$ direction, these chains are separated by corner sharing $\mathrm{GeO}_{4}$ tetrahedra that are also stacked along $c$ (see Fig. 1). Below $T \approx 35 \mathrm{~K}$, short-range ordering was observed and two different magnetic phases with incommensurate longrange order were reported for $\mathrm{NaFeGe}_{2} \mathrm{O}_{6}$ with transition temperatures at $T_{\mathrm{N}} \approx 13 \mathrm{~K}$ and $T_{\mathrm{MF}} \approx 11.6 \mathrm{~K}[33$ 36]. First at $T_{\mathrm{N}}$ an incommensurate spin-density wave (SDW) evolves with moments pointing roughly along $a$-direction 33 and below $T_{\mathrm{MF}}$, spins form a chiral spin structure with moments lying within the ac plane [34, 35]. A small $b$ component of the chiral structure was controversially discussed [34, 35, and in 35] only a single transition is observed. Simultaneous to the onset of the chiral spin arrangement, a ferroelectric polarization $\left(\boldsymbol{P} \approx 32 \mu \mathrm{C} / \mathrm{m}^{2}\right)$ develops 29,36 . The ferroelectric polarization is confined to the $a c$-plane as the given symmetry forbids a non-zero component of the polarization along $b$ [33], see section III, but symmetry allows for a finite $b$-component of the chiral spin structure.

The simplicity of the phase diagram makes $\mathrm{NaFeGe}_{2} \mathrm{O}_{6}$ an ideal candidate to investigate multiferroic domain dynamics. In the following sections we will first discuss the presence of the magnetic $b$ component utilizing neutron polarization analysis. Subsequently we will report on our investigations of multiferroic domain inversion in $\mathrm{NaFeGe}_{2} \mathrm{O}_{6}$. Finally we will discuss the measured spinwave velocity, which is proposed to limit the inversion speed of multiferroic domains.

\section{EXPERIMENTAL METHODS}

A detailed description of the $\mathrm{NaFeGe}_{2} \mathrm{O}_{6}$ single crystal growth by the top seeding technique can be found in Ref. 36. For our measurements two single crystals (SI and SII) were prepared and had been characterized by susceptibility measurements utilizing a commercial superconducting quantum interference device (SQUID) magnetometer. For both samples the transition temperatures are slightly reduced to $T_{\mathrm{N}} \approx 12.5 \mathrm{~K}$ and $T_{\mathrm{MF}} \approx 11.1 \mathrm{~K}$ with respect to literature [36]. In order to apply electric fields to the system, both samples were mounted between aluminium plates, which can be connected to a high voltage setup. As the ferroelectric polarization is largest along the $a$ direction [36, the plate normal was oriented parallel to it. The sample surface was thinly coated with silver paste
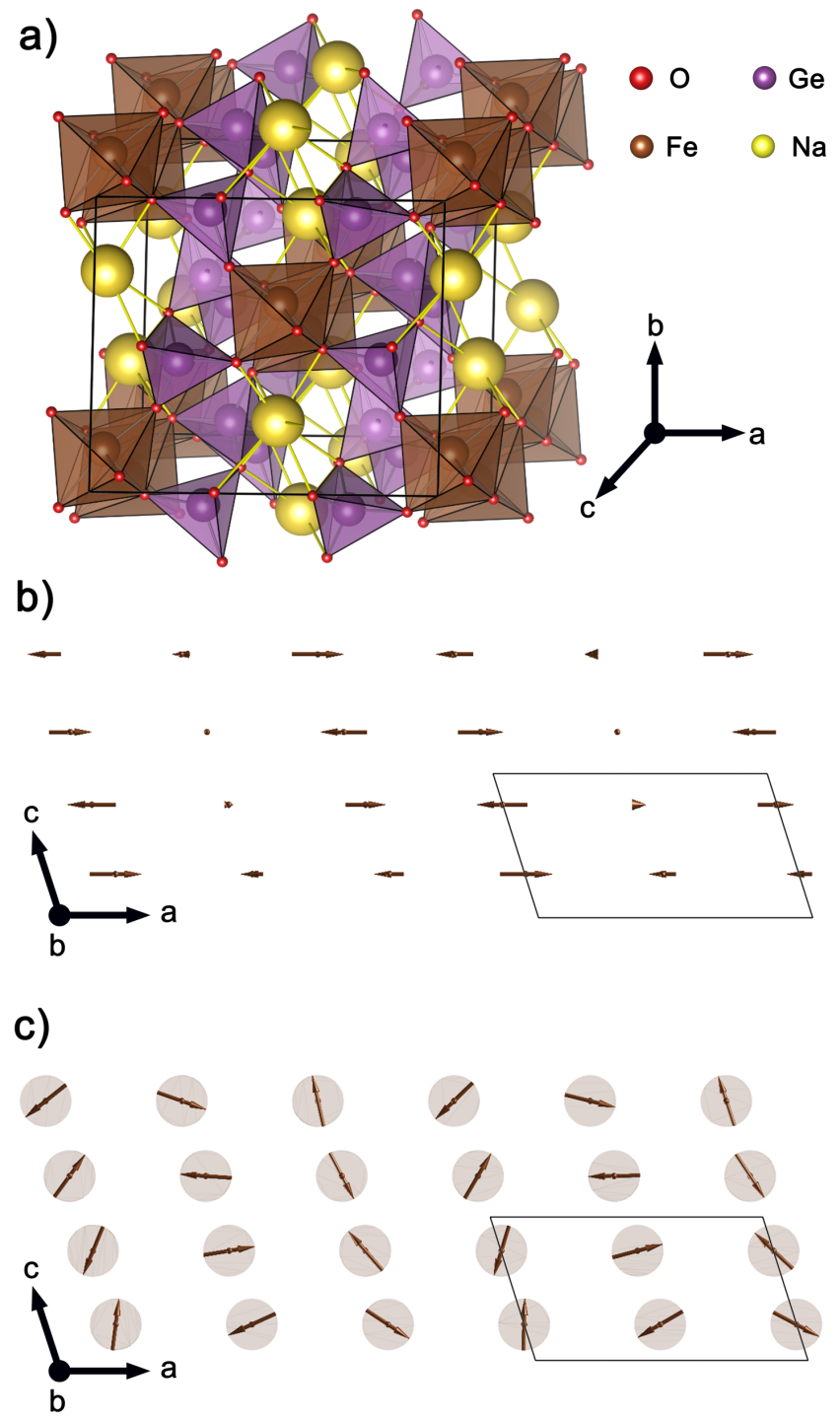

FIG. 1. The nuclear structure (with data from Ref. 35) is shown in panel a) by utilizing the crystallographic visualization software VESTA3 38. Both panels b) and c) sketch the magnetic structure of the intermediate SDW phase and of the low temperature spin-spiral phase, respectively.

for optimized electric contact and the aluminum plates were tightened together by insulating polytetrafluorethylene (PTFE) screws (see Ref. 20). The plates that are cramping the respective sample were connected to sample holders defining the scattering geometry $\left(\begin{array}{lll}1 & 0 & 0\end{array}\right) /\left(\begin{array}{lll}0 & 0\end{array}\right.$ 1) for sample SI and $\left(\begin{array}{lll}1 & 0 & 0\end{array}\right) /\left(\begin{array}{lll}0 & 1 & 0\end{array}\right)$ for sample SII.

Neutron polarization analysis senses the different components of the magnetic structure and the sign of the vector chirality. The respective experiments were performed at the cold three-axes spectrometer IN12 (located at the Institut Laue-Langevin) and at the cold three-axes spectrometer 4F1 (located at the Laboratoire Léon Brillouin).

At IN12, a horizontally and vertically focussing pyrolytic graphite (002) monochromator supplied $\lambda=3 \AA$ and a supermirror cavity provided a highly polarized neu- 
tron beam (flipping ratio of about $\mathrm{FR} \approx 24$ ). Higher order contaminations were suppressed by a velocity selector. The IN12 spectrometer was also equipped with a Helmholtz-coil setup in order to define the guide-field direction at the sample position, and spin-flippers before and after the sample position enabled longitudinal polarization analysis. A curved Heusler (111) analyzer selected the final beam polarization. A conventional high-voltage generator (FUG HCP 14-3500) was deployed for measurements with quasistatic electric fields $(|U| \leq 3.5 \mathrm{kV})$ but a stroboscopic high-voltage setup was used for the time-resolved investigation of the multiferroic domain relaxation. This setup contains two highvoltage modules (ISEG BPP4W and ISEG BPN4W) providing high voltage $(|U| \leq 4 \mathrm{kV})$ with opposite polarity and a fast MOSFET array (BEHLKE HTS-111), which is capable to switch the high voltage output polarity with $50 \mu \mathrm{s}$ [20]. A multichannel data collector (MESYTEC MCPD-8) records all detector events as well as a synchronization signal, when the polarity of the applied electric-field is switched. All events are recorded together with a timestamp, which allows to periodically switch the field, while summing the detected neutrons in time bins with respect to the moment when the field is reversed. This stroboscopic method thus enables one to investigate very fast relaxation processes, because the width of each time bin can be arbitrarily selected, while repeating the switching periodically yields the required statistics. The time resolution is limited by the finite spreads of neutron velocities and path lengths. Ref. 20 gives a detailed description of this setup and of the stroboscopic technique.

For long relaxation times above several minutes, the counting statistics obtained during a single switching period is sufficient and does not require the stroboscopic setup. The respective measurements of long relaxation times were performed at the cold three-axes spectrometer $4 \mathrm{~F} 1$. A double pyrolytic graphite monochromator set-up supplied $\lambda=4.2 \AA$ and a supermirror bender polarized the neutron beam (flipping ratio of about $\mathrm{FR} \approx 50$ ). For polarization analysis a MuPad system [39] and a horizontally curved Heusler analyzer were installed. In order to measure the spin-wave velocity, a polarized beam is not required and the respective experiment was conducted at the $4 \mathrm{~F} 2$ spectrometer. The double pyrolytic-graphite monochromator provided an unpolarized neutron beam with $\lambda=4.05 \AA$ and the pyrolytic-graphite analyzer enables inelastic measurements, for which a cooled Be filter was additionally inserted.

\section{MAGNETIC STRUCTURE}

The magnetic structure of $\mathrm{NaFeGe}_{2} \mathrm{O}_{6}$ was not fully characterized, as a $b$ component of the chiral spin structure was controversially discussed [34, 35] and as the determination of the intermediate structure is based on neutron powder diffraction experiments [33, 34]. Neu- tron polarization analysis on single crystals permits a precise separation of magnetic components and unambiguous identification of chiral signatures.

The symmetry conditions of incommensurate magnetic order with a propagation vector $\boldsymbol{k}=\left(k_{h} 0 k_{l}\right)$ have been discussed for $\mathrm{NaFeGe}_{2} \mathrm{O}_{6}$ in reference [33] they are furthermore identical to those in multiferroic $\mathrm{LiFe}\left(\mathrm{WO}_{4}\right)_{2}$ 40, 41. In space group $C 2 / c$ only one symmetry element has to be considered, the glide mirror plane $c$, which transforms $\left(m_{x}, m_{y}, m_{z}\right)$ into $\left(-m_{x}, m_{y},-m_{z}\right)$. Therefore, it is obvious that magnetic $x, z$ and $y$ components are separated. The character concerning this $c$ element is plus or minus 1 up to the phase correction. The inverse of this phase enters the basis vector components for the second site. In the magnetic superspace group formalism all magnetic moments are developed in cosine and sine series starting from the atoms in the primitive unit cell. Here we have two magnetic ions in the unit cell and the coefficients of these two are not independent but determined through the condition that the moment at $-\mathbf{r}$ and $-x_{4}$ (the argument of the modulation functions) is identical to the moment at $\mathbf{r}$ and $x_{4}$, which is just the inversion symmetry [42. Therefore, only one set of cosine and sine functions is required in the harmonic description. For one-dimensional irreducible representations, as it is the case here, there is a one-to-one correspondence between irreducible representation and magnetic superspace group formalisms, but the representation analysis leaves the phases undefined, while magnetic superspace group analysis fixes these phases leaving only three free parameters for each of the magnetic modes 42. The symmetry of the two different incommensurate magnetic modes is given in Table I.

The magnetic superspace group analysis explains that a multiferroic cycloid cannot be obtained with a single symmetry mode, as the inversion symmetry is not broken 42. One may also argue that in each of the two modes the $x$ and $z$ components are modulated in phase, while $x, z$ and $y$ components have a different character between the two sites so that any spin-current contributions cancel out, as it is also the case in the intermediate phase in $\mathrm{MnWO}_{4}$ [4]. In order to generate cycloidal order one needs to couple two magnetic modes with a phase shift of $\left(\frac{\pi}{2}+n \pi\right)$ by either combining the two different magnetic modes or by combining twice the same mode with the phase shift. In the former case the glide-mirror symmetry is lost and ferroelectric order appears along the $b$ direction as in $\mathrm{LiFe}\left(\mathrm{WO}_{4}\right)_{2}$ [40, 41, while in the latter case the mirror plane persists and enforces ferroelectric polarization in the $a, c$ plane [33. This is the situation in the multiferroic phase of $\mathrm{NaFeGe}_{2} \mathrm{O}_{6}$.

For neutron polarization analysis it is convenient to define a right-handed coordinate system, where the $x$ direction is set parallel to the scattering vector $\boldsymbol{Q}, y$ is perpendicular to $x$ but confined to the scattering plane and $z$ is perpendicular to both, $x$ and $y$. Magnetic scattering will only arise from magnetic components perpendicular to the scattering vector and spin-flip (SF) pro- 
TABLE I. Symmetry conditions for the two incommensurate magnetic modes that can appear for a propagation vector $\boldsymbol{k}=\left(k_{h} 0 k_{l}\right)$ in space group $C 2 / c$ with a single magnetic ion at $(0,0.904,0.25)$. The conditions are defined by the irreducible representation $\Gamma_{1}$ and $\Gamma_{2}$ and the corresponding basis vectors for the two Fe sites are given with $a=e^{i 2 \pi \delta \mathbf{r q}_{\mathbf{i n c}}}$. Here $\delta \mathbf{r}=\mathbf{r}_{2}-\mathbf{r}_{1}$ and $a^{*}=1 / a$ is the complex conjugate of $a$. Representation analysis and superspace group formalism are equivalent in the case of $\mathrm{NaFeGe}_{2} \mathrm{O}_{6}$, but the superspace group formalism fixes the phases between modulations so that for each of the two modes the magnetic structure is described by only three parameters corresponding to either cosine or sine modulations. The resulting superspace groups for a single mode are given in the last column.

\begin{tabular}{c|cc|cc|ccc}
\hline \hline$\Gamma$ & 1 & $c$ & $(x, y, z)$ & $(x, \bar{y}, z+1 / 2)$ & coefficients & character & superspace group \\
\hline$\Gamma_{1}$ & 1 & $-a$ & $(u, v, w)$ & $a^{*}(u,-v, w)$ & $\operatorname{Re}(u) \operatorname{Im}(v) \operatorname{Re}(w)$ & $\cos / \sin / \cos$ & $C 2 / c 1^{\prime}(\alpha 0 \gamma) 0 s s$ \\
$\Gamma_{2}$ & 1 & $a$ & $(u, v, w)$ & $a^{*}(-u, v,-w)$ & $\operatorname{Im}(u) \operatorname{Re}(v) \operatorname{Im}(w)$ & $\sin / \cos / \sin$ & $C 2 / c 1^{\prime}(\alpha 0 \gamma) 00 s$ \\
\hline \hline
\end{tabular}

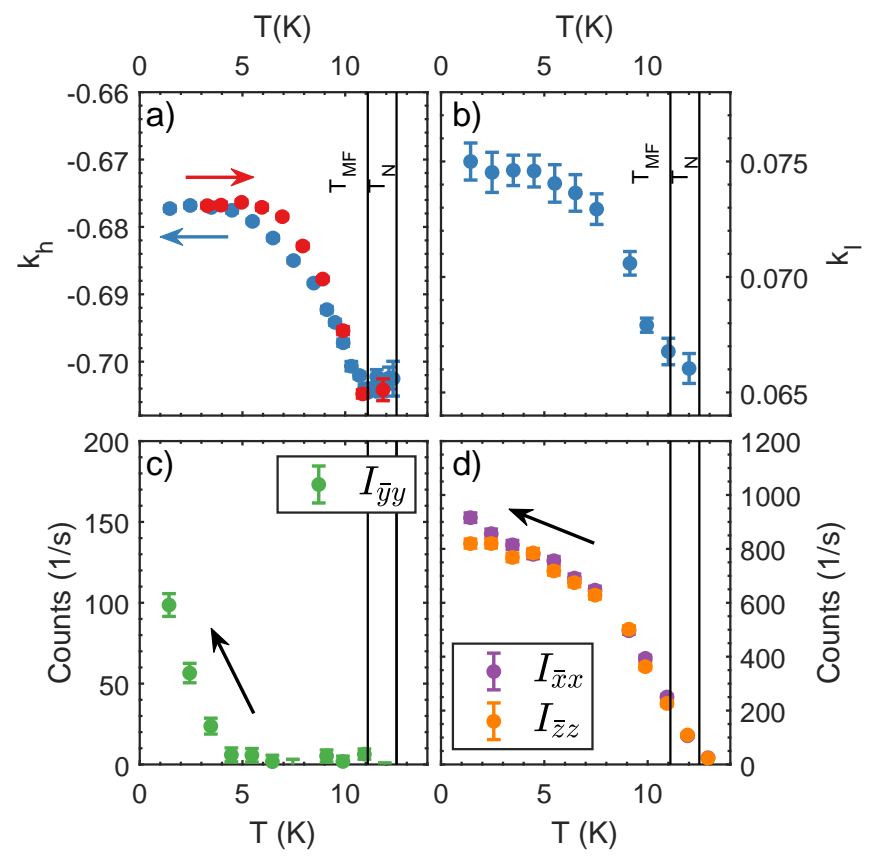

FIG. 2. Panels a) and b) display the temperature dependent components $k_{h}$ and $k_{l}$ of the propagation vector $\boldsymbol{k}=\left(k_{h} 0 k_{l}\right)$, respectively. The blue and red data correspond to cooling and heating sequences, respectively. In c) and d) the peak intensity of reflection $\boldsymbol{Q}=\left(\begin{array}{lll}0.678 & 0 & -1.075\end{array}\right)$ is plotted for all $\mathrm{SF}$ channels as function of temperature. All intensities were corrected for the finite flipping ratio and all measurements were performed on sample SI.

cesses necessitate a component of the magnetization perpendicular to the direction of the incoming beam polarization. In contrast, non-spin-flip (NSF) processes only sense components that are aligned parallel or antiparallel to the incoming neutron beam polarization. During the first part of the allocated beamtime at IN12, sample SI with scattering geometry $\left(\begin{array}{lll}1 & 0 & 0\end{array}\right) /\left(\begin{array}{lll}0 & 0 & 1\end{array}\right)$ was mounted. Both components of the incommensurate propagation vector $\boldsymbol{k}=\left(\begin{array}{lll}k_{h} & 0 & k_{l}\end{array}\right)$ could be traced as a function of temperature. For the respective measurements, $Q_{h}$ and $Q_{l}$ scans were executed across the magnetic reflection $\boldsymbol{Q}=\left(\begin{array}{lll}0.678 & 0 & -1.075\end{array}\right)$ and each $Q$ scan was fitted by a Gaussian function. The observed peak center values were considered for defining the scan of the following step in temperature. Fig. 2 a) and b) display the obtained temperature dependent values for $k_{h}$ and $k_{l}$, and it can be seen that both components vary with temperature but lock in a constant value at $T \approx 5 \mathrm{~K}$. The results qualitatively agree with the reported temperature dependence of the incommensurate propagation vector in Ref. 35.

The used scattering geometry entails, that the SF channels are described by $I_{\bar{y} y} \propto M_{b} M_{b}^{*}$ and $I_{\bar{z} z} \propto$ $M_{a c} M_{a c}^{*} \sin ^{2}(\alpha)$ with $M_{b}$ and $M_{a c}$ being the complex Fourier components of the magnetization along $b$ direction and within the $a c$ plane, respectively, and $\sin ^{2}(\alpha)$ being the geometry factor. $a$ and $a^{*}$ are not parallel in the monoclinic system, but in the used scattering geometry both are lying within the scattering plane. The same holds for $c$ and $c^{*}$. Due to the incommensurability of the magnetic structure, no nuclear signal contributes to the measured intensity. The SF channels $I_{\bar{x} x}$ and $I_{x \bar{x}}$ are described by $\boldsymbol{M}_{\perp} \boldsymbol{M}_{\perp}^{*} \pm i\left(\boldsymbol{M}_{\perp} \times \boldsymbol{M}_{\perp}^{*}\right)_{x}$ but as the scattering vector lies within the basal plane of the chiral spin structure the chiral term vanishes. The intensity of all three SF-channels are plotted in Fig. 2 c) and d). It can be clearly seen in c) that $I_{\bar{y} y}$ exhibits finite intensity only below $T \approx 5 \mathrm{~K}$ thus indicating the development of a finite $b$ component of the magnetic structure within the multiferroic phase of $\mathrm{NaFeGe}_{2} \mathrm{O}_{6}$, but exclusively at low temperature. The onset of this component coincides with the lock-in temperature of the incommensurate propagation vector. Fig. 2 d) shows that below the first transition at $T_{\mathrm{N}}$, the total magnetic scattering signal $I_{\bar{x} x}$ initially features the same amplitude as $I_{\bar{z} z}$, which senses the magnetic moments within the $a c$ plane. This confirms that the magnetic structure is first confined to the $a c$ plane until below $T \approx 5 \mathrm{~K}$ the discrepancy between $I_{\bar{z} z}$ and $I_{\bar{x} x}$ also indicates the evolving $b$ component of the magnetic structure.

Sample SII was mounted with scattering geometry (1 $\left.\begin{array}{ll}0 & 0\end{array}\right) /\left(\begin{array}{lll}0 & 1 & 0\end{array}\right)$. In this configuration the SF-channels $I_{\bar{y} y}$ and $I_{\bar{z} z}$ sense the magnetization along $c$ direction and within the $a^{*}, b$ plane, respectively. The magnetic reflection $\boldsymbol{Q}=\left(\begin{array}{lll}0.32 & 3 & 0.08\end{array}\right)$ is not directly accessible within the given scattering plane but the finite $l$ value can be reached by tilting the goniometer by $\approx 3^{\circ}$, which does not have significant impact on the analysis of respective SF and NSF channels. The temperature dependence of both SF channels is plotted in Fig. 3 a) and it is visible that 


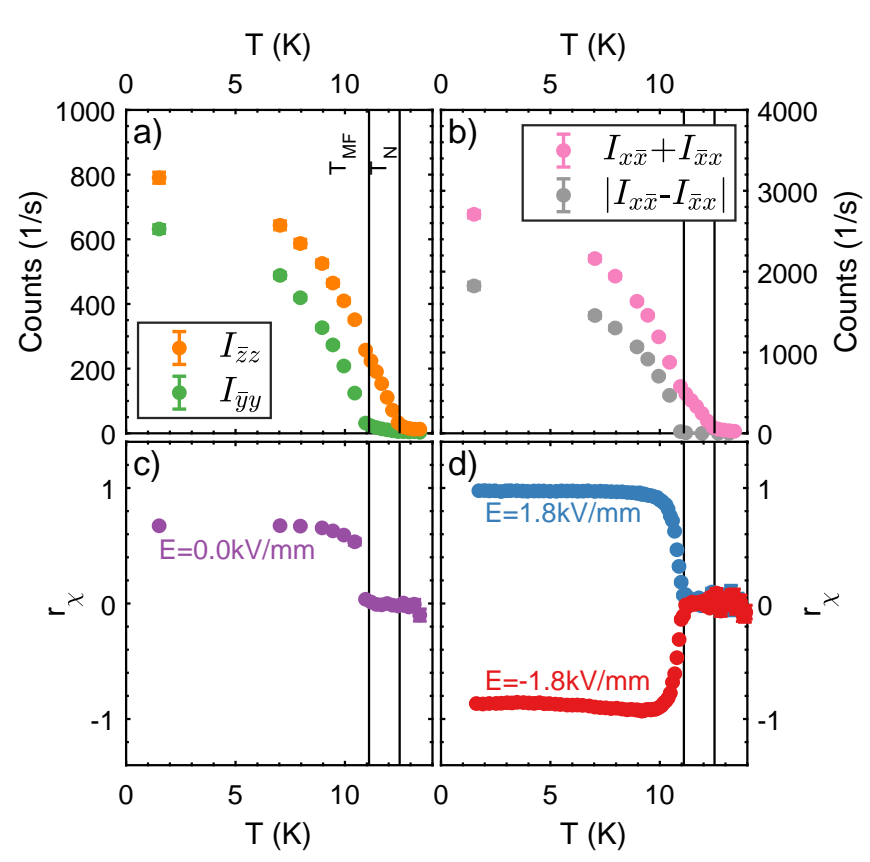

FIG. 3. Polarized neutron scattering in $\left(\begin{array}{lll}1 & 0 & 0\end{array}\right) /\left(\begin{array}{lll}0 & 1 & 0\end{array}\right)$ geometry. The temperature dependence of SF channels measured at $\boldsymbol{Q}=(0.3230 .08)$ is shown in a) and b). The $Q$-space position was adjusted with the determined temperature dependent values of the incommensurate propagation vector (see Fig. 2a) and b)). In both panels c) and d) the chiral ratio is plotted as a function of temperature and for different applied electric field amplitudes.

the $c$ component of the magnetic structure mainly develops below the second transition $T_{\mathrm{MF}}$ but nevertheless finite intensity and thus a non-zero component along $c$ is also present in the intermediate phase. The chiral spin structure in the multiferroic phase thus exhibits moments in the $a c$ plane with an additional $b$ component, which develops below $T \approx 5 \mathrm{~K}$. In the intermediate phase between $T_{\mathrm{N}}=12.5 \mathrm{~K}$ and $T_{\mathrm{MF}}=11.1 \mathrm{~K}$ the SF channel $I_{\bar{z} z}$ possesses extensive intensity compared to $I_{\bar{y} y}$ and as the angle between $\boldsymbol{Q}$ and $b$ amounts approximately $6.9^{\circ}$, the SF channel $I_{\bar{z} z}$ essentially senses the component parallel to $a^{*}$. With results from both scattering geometries it can be concluded that the SDW structure exhibits moments $\boldsymbol{m}$ that are lying essentially in the $a, c$ plane with $\measuredangle\left(\boldsymbol{m}, \boldsymbol{a}^{*}\right) \approx 7^{\circ}$.

The scattering vector in the scattering configuration of sample SII is almost perpendicular to the basal plane of the chiral structure. Therefore, the chiral term $\pm i\left(\boldsymbol{M}_{\perp} \times\right.$ $\left.\boldsymbol{M}_{\perp}^{*}\right)_{x}$ is finite and contributes to both SF channels $I_{x \bar{x}}$ and $I_{\bar{x} x}$ with a different sign. Hence, the sum and the difference of $I_{x \bar{x}}$ and $I_{\bar{x} x}$ are given by $2 \boldsymbol{M}_{\perp} \boldsymbol{M}_{\perp}^{*}$ and by the absolute value of $-2 i\left(\boldsymbol{M}_{\perp} \times \boldsymbol{M}_{\perp}^{*}\right)_{x}$, respectively. Both, the sum and the difference of $\bar{I}_{x \bar{x}}$ and $I_{\bar{x} x}$ are plotted in Fig. $3 \mathrm{~b}$ ) as a function of temperature and it can be seen that the chiral signal develops only below $T_{\mathrm{MF}}$ within the multiferroic phase. The chiral ratio is defined
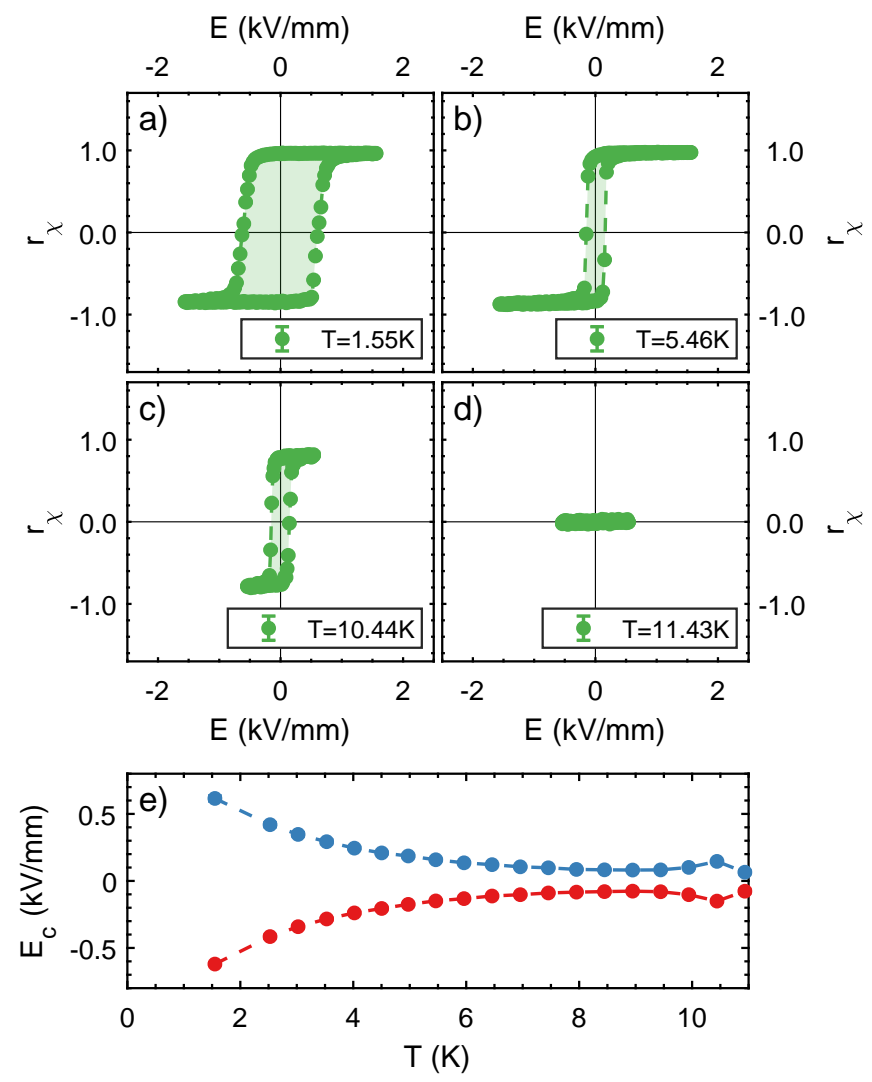

FIG. 4. All recorded hysteresis loops of the chiral ratio shown in panels a)-d) were obtained by driving the applied external electric field slowly (with a period duration of about $45 \mathrm{~min}$ ) between the two polarities. Both slopes of each hysteresis loop were fitted by a hyperbolic tangent in order to estimate the values of respective coercive fields, which are plotted in e).

as $r_{\chi}=\left(I_{x \bar{x}}-I_{\bar{x} x}\right) /\left(I_{x \bar{x}}+I_{\bar{x} x}\right)$ and for a mono domain sample it corresponds to $\pm i\left(\boldsymbol{M}_{\perp} \times \boldsymbol{M}_{\perp}^{*}\right)_{x} /\left|\boldsymbol{M}_{\perp}\right|^{2}$, the ratio of the chiral part with respect to the overall magnetic scattering contribution. The chiral ratio amounts to \pm 1 only for a completely poled multiferroic state with ideal geometry. However, as the scattering vector $\boldsymbol{Q}$ is not perfectly oriented perpendicular to the rotation plane of spins, and due to ellipticity the chiral ratio remains below one. In Fig. 3 c) the chiral ratio is plotted and it can be seen that even in the absence of an applied electric field, the chiral ratio possesses a finite value within the multiferroic phase and thus indicates an unbalanced domain distribution of multiferroic domains [22, 41]. The quasistatic control of the chiral handedness and the multiferroic domain population by external electric fields is discussed in the following section. 


\section{QUASISTATIC CONTROL OF MULTIFERROIC DOMAINS}

A static electric field with $|E|=1.8 \mathrm{kV} / \mathrm{mm}$ was applied, while cooling the sample. The chiral ratio was measured simultaneously and its temperature dependence is shown in Fig. 3 d) for both field polarities. It can be seen that the chiral ratio reaches the maximum values of $\approx \pm 1$ almost immediately below $T_{\mathrm{MF}}$, which indicates a completely poled multiferroic domain distribution and a circular cycloid. This result illustrates the power of polarized neutron diffraction to solve chiral structures, as only the combination of twice the same magnetic mode can cause a chiral signal in this configuration. The chiral phase in $\mathrm{NaFeG}_{2} \mathrm{O}_{6}$ thus must arise from a single irreducible representation similar to $\mathrm{NaFeSi}_{2} \mathrm{O}_{6}$ [31 but the orientation of the propagation vector differs. An opposite field polarity entails a reversed chiral ratio and an equivalent temperature dependence documenting the controllability of multiferroic domains with applied electric fields in $\mathrm{NaFeGe}_{2} \mathrm{O}_{6}$. The behavior does not significantly change when lowering the field amplitude to $|E|=1.2 \mathrm{kV} / \mathrm{mm}$ and $|E|=0.6 \mathrm{kV} / \mathrm{mm}$. In order to quantify the respective values and the temperature dependence of the electric-field amplitude that is needed to reverse the chirality, quasistatic hysteresis loops have been recorded.

Fig. 4 displays hysteresis loops of the chiral ratio for different temperatures. The electric field was driven quasistatically between the two polarities corresponding to a period of about 45 minutes for the entire hysteresis loops. The sign of the vector chirality is invertible down to the lowest temperature at $T=1.55 \mathrm{~K}$. While approaching the transition to the intermediate phase, the saturation value of the chiral ratio shrinks slightly and abruptly vanishes, when heating above the multiferroic transition. This was also observed by the cooling sequences with applied static fields (see Fig. 3 d)). The coercive fields that are needed to invert the sign of the chiral ratio in positive or negative direction were determined by fitting both slopes of the hysteresis loops with a hyperbolic tangent. The obtained coercive fields are plotted in Fig. 4 e) as a function of temperature. The coercive fields exhibit a symmetric temperature dependence with the smallest values close to the multiferroic transition.

\section{MULTIFERROIC DOMAIN RELAXATION}

In order to investigate the relaxation of multiferroic domains in $\mathrm{NaFeGe}_{2} \mathrm{O}_{6}$, while triggering the inversion of domains by external electric fields, the stroboscopic setup was installed at IN12 (see section III). The switching curves of the chiral ratio were recorded for different temperatures and a variety of electric-field amplitudes on sample SII. Fig. 5 displays four exemplary switching curves and it can be seen by comparing the pan-
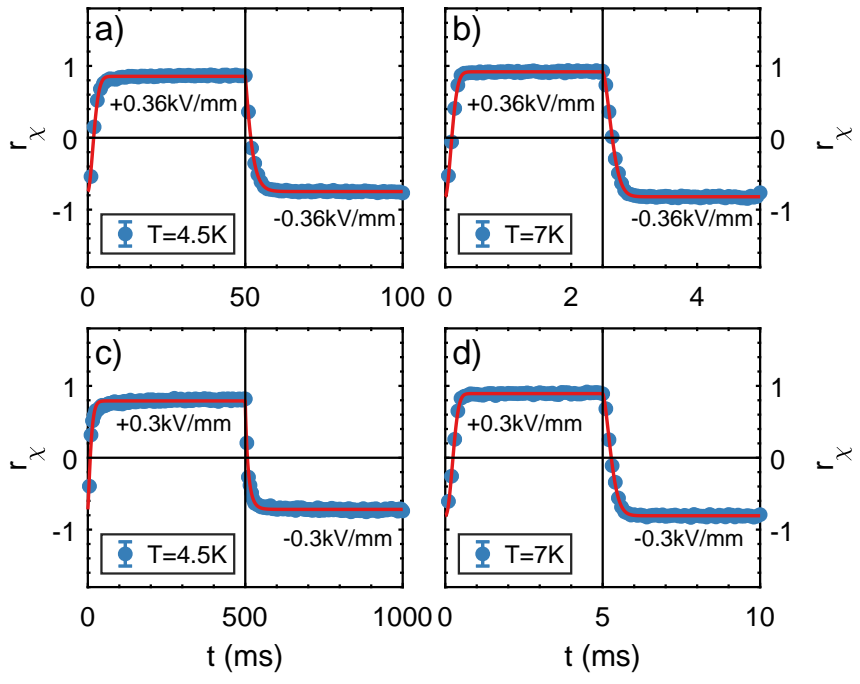

FIG. 5. All displayed switching curves in a)-d) were recorded by utilizing the stroboscopic method at IN12 and by measuring on sample SII. The blue data points refer to the recorded chiral ratio and the red line corresponds to the fit. The time dependence of the applied electric field follows a rectangular shape with inversion at the beginning and in the middle of each panel.
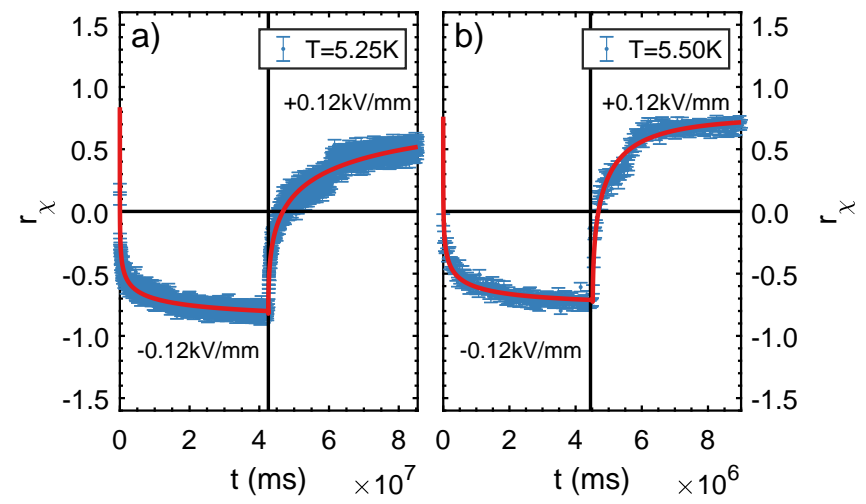

FIG. 6. At $4 \mathrm{~F} 1$ the relaxation processes of the chiral ratio, which is plotted in a and b), were recorded by counting both SF channels $I_{x \bar{x}}$ and $I_{\bar{x} x}$ as a function of time. The electric field $(|E|=0.12 \mathrm{kV} / \mathrm{mm})$ was manually switched after the saturation of the chiral ratio for the respective handedness was reached. For both curves sample SII was measured.

els horizontally that the relaxation time significantly decreases, when increasing the temperature. Moreover, the vertical arrangement of panels in Fig. 5 displays the field dependence of the relaxation processes. It can be seen that the electric-field strength at fixed temperature also strongly influences the relaxation processes. For all recorded curves, there is no asymmetric relaxation behavior concerning the electric field polarity.

The relaxation process can be described by an expo- 
nential relaxation in both directions [20]:

$$
\begin{aligned}
& r_{\chi}(t)=r_{a}-\left(r_{a}-r_{b}\right) \exp \left(-\left(\frac{t}{\tau_{a}}\right)^{b_{1}}\right) \\
& r_{\chi}(t)=r_{b}-\left(r_{b}-r_{a}\right) \exp \left(-\left(\frac{t-t_{1 / 2}}{\tau_{b}}\right)^{b_{2}}\right)
\end{aligned}
$$

Both parameters $\tau_{a}$ and $\tau_{b}$ are the characteristic relaxation times describing the inversion of the chiral ratio from $r_{a}$ to $r_{b}$ and from $r_{b}$ to $r_{a}$, respectively. Here, $t_{1 / 2}$ accounts for the reversal of the electric field after half the period. Considering the Ishibashi-Takagi theory, which is based on the Avrami-model, the stretching exponents $b_{1}$ and $b_{2}$ describe the dimensionality of the domain wall motion 4448 . Both stretching exponents vary between 1 and 2.5 thus indicating low-dimensional domain growth. Approaching $T_{\mathrm{N}}$ or enhancing the field amplitude result in larger stretching exponents suggesting a higher growth dimensionality or possibly additional nucleation processes. A similar behavior was observed for $\mathrm{TbMnO}_{3}$ [20].

The investigation of long relaxation times $(>1 \mathrm{~min})$ does not require the stroboscopic setup, as sufficient statistics are reached during a single switching period. The respective measurements were performed at $4 \mathrm{~F} 1$. Two switching curves are exemplarily shown in Fig. 6 for an electric-field amplitude of $|E|=0.12 \mathrm{kV} / \mathrm{mm}$. Although the experiment was performed on the same sample SII, the curves exhibit an asymmetric relaxation behavior contrary to the recorded curves from the IN12 experiment. The system was short circuited at room temperature and during the cooling procedure but nevertheless this asymmetry can be due to residual frozen-in charges or from effective pinning at defects, which provoke a preferred sign of the vector chirality in the multiferroic phase 22]. Moreover, both curves possess a kink after the second field inversion, which can be related to a time-delayed second nucleation process. However, this effect was resolvable only for the several orders of magnitude slower relaxation processes that were investigated during the $4 \mathrm{~F} 1$ beamtime.

Fig. 7 displays the fitted relaxation times $\tau_{a}$ and $\tau_{b}$ (see equation 1 and 2 as a function of inverse temperature and of inverse field with a logarithmic scaling. Up and down arrows mark the fitted relaxation time from IN12 data for switching the electric field to positive and negative values, respectively. The crosses in a) and b) mark the fitted relaxation times, which have been obtained from the $4 \mathrm{~F} 1$ experiment. Combining both experiments at IN12 and 4F1 it is possible to follow the multiferroic relaxation over 7 orders of magnitude in time as a function of temperature and electric field.

The multiferroic domain relaxation in $\mathrm{TbMnO}_{3}$ [20] follows a combined Arrhenius-Merz law as a function of temperature and electric-field:

$$
\tau(E, T)=\tau^{*} \exp \left(\frac{A_{0} T_{r}}{E T}\right) \text { with } T_{r}=\frac{T_{\mathrm{MF}}-T}{T_{\mathrm{MF}}}
$$
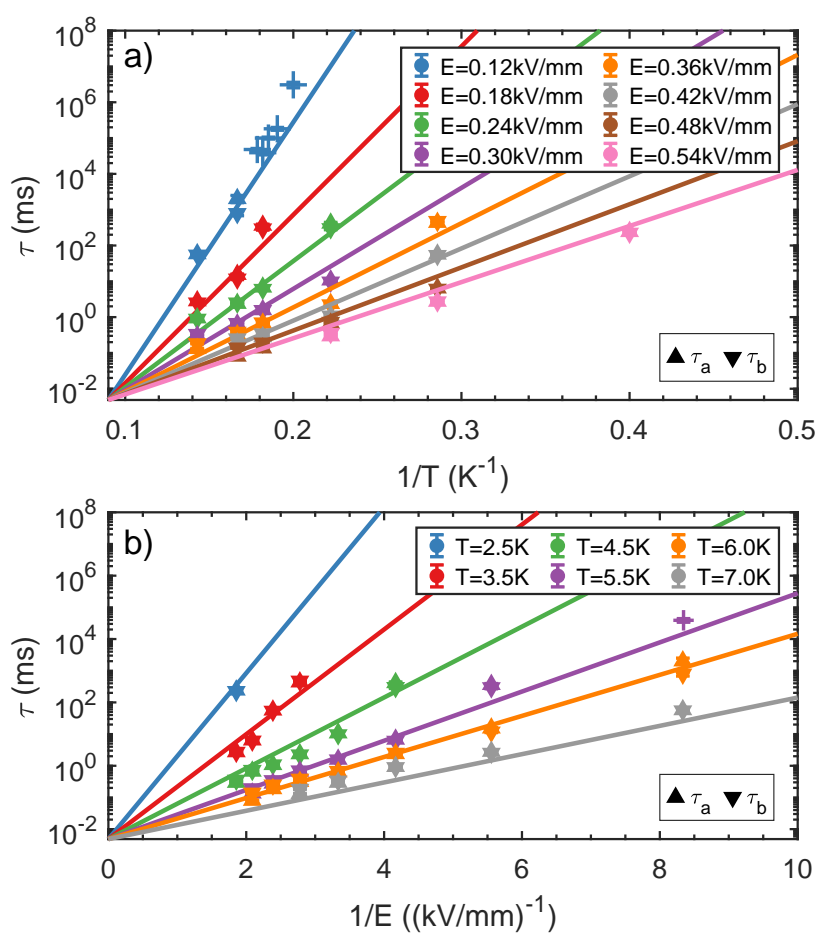

FIG. 7. Panels a) and b) display the temperature and electricfield dependent relaxation times, respectively. Up and down triangles refer to $\tau_{a}$ and $\tau_{b}$ from IN12 data, whereas crosses correspond to $\tau_{a}$ from the recorded $4 \mathrm{~F} 1$ data. The whole IN12 data set was fitted simultaneously by the combined ArrheniusMerz law (see equation 3) and the plotted colored solid lines refer to the fit results shown for fixed electric fields in a) or fixed temperatures in $\mathrm{b}$ ). The $4 \mathrm{~F} 1$ data were not included to the fit.

Here $\tau^{*}$ denotes the fastest possible relaxation time that can be reached at the multiferroic phase transition $T_{\mathrm{MF}}=T_{\mathrm{N}}$ or at infinitely large fields, and $A_{0}$ is the activation constant. Thus, $\tau^{*}$ and $A_{0}$ are the only two parameters needed to describe the field and temperature dependence of the relaxation. For $\mathrm{TbMnO}_{3}$ the parameters amount to $\tau^{*}=0.72 \mathrm{~ms}$ and $A_{0}=1483 \mathrm{~K} \mathrm{kV} / \mathrm{mm}$ 20]. This combined Arrhenius-Merz relation describes multiferroic relaxation in the E, T range, where it is determined by classical domain-wall motion. In the studied E, $\mathrm{T}$ range, the multiferroic relaxation in $\mathrm{TbMnO}_{3}$ deviates from the combined Arrhenius-Merz law only when approaching the multiferroic transition, as additional nucleation processes seem to accelerate the domain inversion.

For $\mathrm{NaFeGe}_{2} \mathrm{O}_{6}$ all relaxation times obtained from IN12 data were simultaneously fitted with the combined Arrhenius-Merz law. The 4F1 data were excluded from the fit as even a tiny temperature offset between both instruments due to different sample sticks and temperature calibration has significant impact on the fit result. Furthermore, the asymmetry of relaxation and the second nucleation process complicate the precise determination of relaxation times. Nevertheless, the temperature 

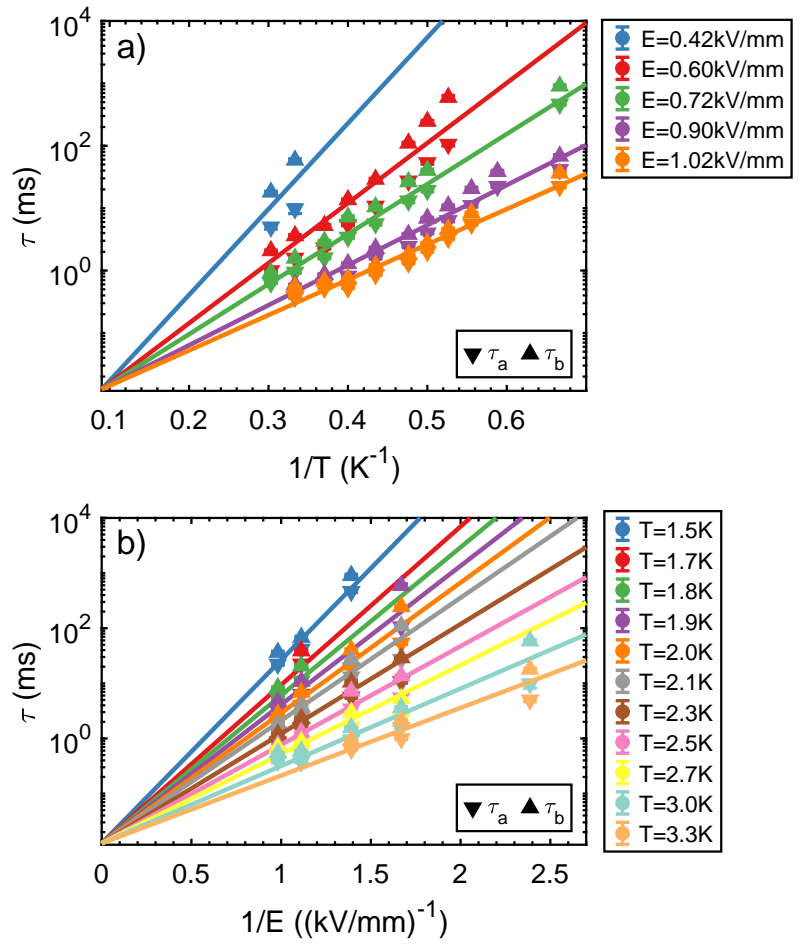

FIG. 8. Additional set of measurements of the relaxation times from a second experiment are displayed in panels a) and b). The combined Arrhenius-Merz law is valid even at very low temperatures. A slightly different activation constant and critical relaxation time with respect to results shown in Fig. 7 arise from a renewed sample setup (see text).

and electric-field dependent relaxation times $\tau_{a}$ from $4 \mathrm{~F} 1$ data are included in Fig. 7 (see crosses). Due to the observed asymmetry for long relaxation times only $\tau_{a}$ is plotted, whereas $\tau_{b}$ is approximately one order of magnitude larger.

Solid lines in Fig. 7 a) and b) correspond to the fit result for different temperatures and different electric-field amplitudes. It is clearly visible that the multiferroic relaxation behavior in $\mathrm{NaFeGe}_{2} \mathrm{O}_{6}$ is also well described by this combined activation law requiring only two parameters. The obtained critical relaxation time $\tau^{*}$ and the activation constant $A_{0}$ amount to $\tau^{*}=0.0049(12) \mathrm{ms}$ and $A_{0}=19.46(88) \mathrm{KkV} / \mathrm{mm}$. During a second experiment at the IN12 spectrometer, the relaxation times at very low temperatures were further studied. Before this beamtime, a tiny piece was cut from sample SII for other measurements and the sample contacts were renewed. With the restored sample setup a slightly different critical relaxation time and activation constant was observed but nevertheless both values $\left(\tau^{*}=0.0125(18) \mathrm{ms}\right.$ and $\left.A_{0}=13.28(34) \mathrm{K} \mathrm{kV} / \mathrm{mm}\right)$ are of the same order of magnitude compared to results from the first experiment. The measured relaxation times as well as the fit are shown in Fig. 8 and it can be seen that even at low temperature the relaxation follows the activation law indicating that no quantum effects dominate the relaxation

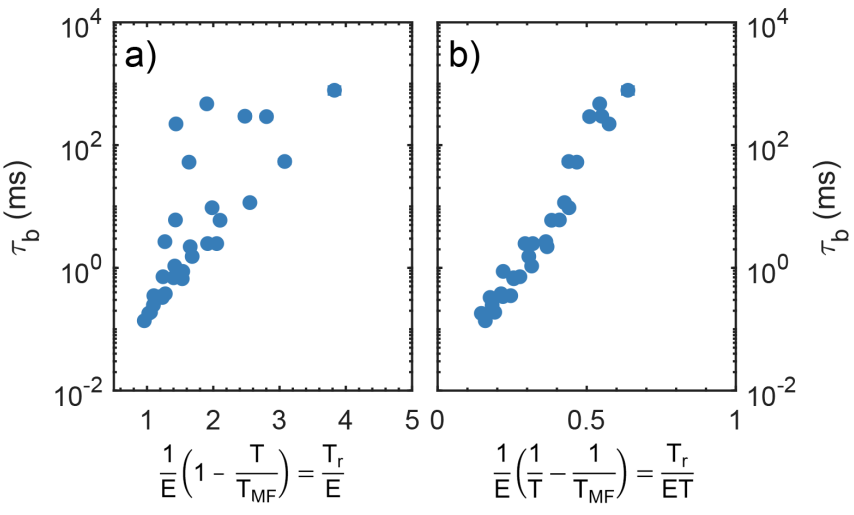

FIG. 9. The different scaling relations for the relaxation times shown in panels a) and b) clearly determine the combined Arrhenius-Merz law to be the correct description of the multiferroic domain inversion. For the comparison only IN12 data from the first experiment (see Fig. 7) were used.

behavior, which would lead to a saturation of the relaxation time. For fields between $E=0.42 \mathrm{kV} / \mathrm{mm}$ and $E=1.02 \mathrm{kV} / \mathrm{mm}$, the domain wall motion in $\mathrm{NaFeGe}_{2} \mathrm{O}_{6}$ remains thus thermally activated and is not driven by domain wall tunneling down to $T=1.5 \mathrm{~K}$. Domain wall tunneling was reported for antiferromagnetic and ferroelectric domain wall motion at low temperature [49, 50]. In $\mathrm{NaFeGe}_{2} \mathrm{O}_{6}$, quantum effects can become relevant at even lower temperature and electric field values.

Some pure ferroelectrics [51, 52] exhibit thermally activated relaxation following a scaling law $\tau(E, T)=$ $\tau^{*} \exp \left(A_{0} T_{r} / E\right)$, in which the relaxation only depends on the ratio $T_{r} / E$. The measurements on multiferroic $\mathrm{TbMnO}_{3}$ [20] do not agree with this but follow a scaling with $T_{r} /(E T)$ as it results from the combined Arrhenius-Merz law. Fig. 9 compares both scaling laws for $\mathrm{NaFeGe}_{2} \mathrm{O}_{6}$ and clearly shows that the combined Arrhenius-Merz law is the appropriate description also for this material.

The relaxation of multiferroic domains differs from the relaxation behavior in conventional ferroelectrics 20, 51, 52. While in a ferroelectric material the nucleation process, the forward growth and the subsequent sideways expansion of domains can equally contribute to the global domain relaxation process and thus render its description complicated [51, 52, multiferroic domain inversion is dominantly determined by the sideways growth of domains [19, 20, 53, 54. Therefore, thermally activated domain wall motion and a combined ArrheniusMerz law 20] describe the multiferroic domain inversion over a wide range of temperatures and fields, spanning over 7 orders of magnitude in time. However, close to the multiferroic transition and for very high fields additional nucleation processes will accelerate the relaxation, and at very low temperatures and electric fields quantum tunneling can become relevant. 

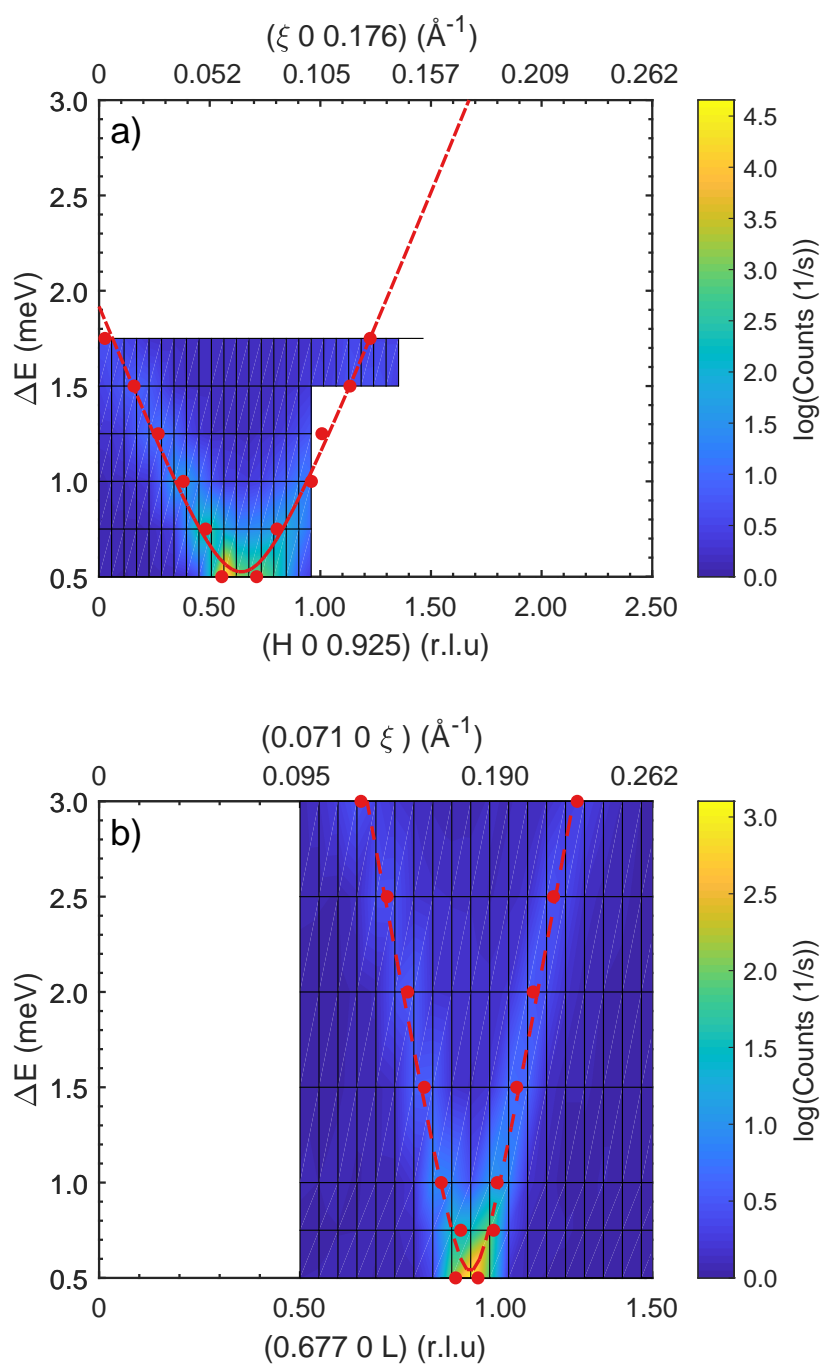

FIG. 10. The plots in a) and b) display the recorded constantenergy scans at $T=3.9 \mathrm{~K}$ around the incommensurate zone

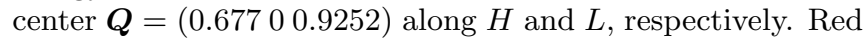
data points refer to the fitted peak maxima and the red lines correspond to fits of the respective dispersion.

\section{SPIN-WAVE VELOCITY}

Since the spin-wave velocity can limit the multiferroic domain wall motion [19, 20] we analyzed the dispersion of acoustic magnon branches at the $4 \mathrm{~F} 2$ triple-axis spectrometer. The respective measurements were carried out on sample SI, which was mounted with scattering geometry $\left(\begin{array}{lll}1 & 0 & 0\end{array}\right) /\left(\begin{array}{lll}0 & 0 & 1\end{array}\right)$. For $\Delta E \geq 0.5 \mathrm{meV}$, constant-energy scans were measured around the incommensurate zone

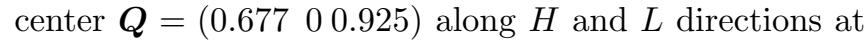
$T=3.9 \mathrm{~K}$. Below $\Delta E=0.5 \mathrm{meV}$ it was not possible to separate the excitation from the elastic line due to the finite resolution.

Fig. 10 maps the measured scattering along both recorded directions. All constant-energy scans were fitted with two Gaussian functions and the obtained peak- center positions of excitations are marked by red data points in a) and b). The dispersion can be approximately described by $\omega(q)=\sqrt{\left(\Delta^{2}+v_{g}^{2} q^{2}\right)}$, with $v_{g}$ the group velocity of the spin wave and $\Delta$ the gap energy. The fits for respective directions (red dashed lines in Fig. 10 yield $v_{g}=662(19) \mathrm{m} / \mathrm{s}$ along $H, v_{g}=1460(36) \mathrm{m} / \mathrm{s}$ along $L$ and a spin gap of approximately $0.53 \mathrm{meV}$ for both directions. The magnon dispersion is thus anisotropic with a steeper slope along the direction of zigzag chains, but this anisotropy is moderate and the material should not be considered as a low-dimensional system. The ferroelectric polarization is predominantly aligned along the $a$ direction [36] and for all respective experiments on the multiferroic domain inversion, the electric field was applied parallel to it. In multiferroics the initial reverted domains extend along the direction of ferroelectric polarization before the subsequent sideways growth dominates the overall relaxation process [19, 20. Considering the determined spin-wave velocities, a single needle-shaped domain in the middle and the geometry of sample SII (for which the relaxation behavior was measured), the fastest possible domain relaxation is of the order of $10^{-3} \mathrm{~ms}$, which agrees with the observed critical relaxation time $\tau^{*}$ (see section V). However, a striped pattern of more than one needle-shaped domain as well as nucleation of additional germs will further reduce the multiferroic relaxation time, because both effects reduce the distances to be overcome by the domain wall motion.

\section{CONCLUSION}

We report investigations on the magnetic structure as well as on the multiferroic domain relaxation in $\mathrm{NaFeGe}_{2} \mathrm{O}_{6}$ utilizing neutron scattering experiments on single crystals. With neutron polarization analysis it is possible to separate the magnetic components of the longrange order in both phases. Thereby we can confirm the proposed incommensurate spin-density wave with moments along the $a$ direction for the intermediate phase and a chiral spin structure with components along $a$ and $c$ below the multiferroic transition. In addition there is a locking-in of the incommensurate propagation vector at $T \approx 5 \mathrm{~K}$ accompanied with the emergence of a $b$-component, whose presence was so far controversially discussed [34, 35].

The multiferroic domain inversion was investigated by quasi-static as well as by time-resolved measurements of the vector chirality. Poling sequences and hysteresis loops confirm the invertibility of its sign and show low coercive fields as a function of temperature. As reported for $\mathrm{TbMnO}_{3}$ 20, time-resolved measurements of the rapidly switched chiral ratio reveal the combined Arrhenius-Merz law to describe the relaxation behavior in $\mathrm{NaFeGe}_{2} \mathrm{O}_{6}$ over at least 7 orders of magnitude in time. Even at low temperatures of the order of $1.5 \mathrm{~K}$ the multiferroic domain inversion is well described by thermally activated 
domain-wall motion.

Since $\mathrm{NaFeGe}_{2} \mathrm{O}_{6}$ allows one to study the multiferroic domain relaxation over a large temperature range (down to $T / T_{\mathrm{MF}} \approx 0.14$ ), we can analyze the scaling of electric field and reduced temperature $T_{\mathrm{r}}$. While a simple scaling $f\left(\frac{T_{r}}{E}\right)$ can be applied to ferroelectrics [51, 52] such scaling can be excluded for the multiferroic domain relaxation in $\mathrm{NaFeGe}_{2} \mathrm{O}_{6}$, which follows the $f\left(\frac{T_{r}}{T E}\right)$ scaling predicted by the Arrhenius-Merz law.

In conclusion, $\mathrm{NaFeGe}_{2} \mathrm{O}_{6}$ exhibits similar to $\mathrm{TbMnO}_{3}$ a simple field and temperature dependence of multiferroic domain inversion that is well described by thermally activated domain-wall motion with an activation field given by $A_{0} \frac{T_{\mathrm{r}}}{T}$.

The neutron scattering data from the IN12 diffractometer are available [55.

\section{ACKNOWLEDGEMENTS}

This work was funded by the Deutsche Forschungsgemeinschaft (DFG, German Research Foundation) Project number 277146847 - CRC 1238, projects A02, B04 and by the Bundesministerium für Bildung und Forschung - Project number 05K19PK1. This work is based upon experiments performed at the IN12 instrument operated by JCNS at the Institute Laue-Langevin (ILL), Grenoble, France. The authors gratefully acknowledge the financial support provided by JCNS to perform the neutron scattering measurements at the at the Institute Laue-Langevin (ILL), Grenoble, France.
[1] N. Jones, "The information factories," Nature 561, 163 (2018).

[2] J. Puebla, J. Kim, K. Kondou, and Y. Otani, "Spintronic devices for energy-efficient data storage and energy harvesting," Commun Mater 1, 24 (2020).

[3] L. Ceze, J. Nivala, and K. Strauss, "Molecular digital data storage using DNA," Nature Reviews Genetics 20, 456 (2019)

[4] K. N. Lin, K. Volkel, J. M. Tuck, and A. J. Keung, "Dynamic and scalable DNA-based information storage," Nature Communications 11, 2981 (2020).

[5] M. Wuttig and N. Yamada, "Phase-change materials for rewriteable data storage," Nature Materials 6, 824 (2007)

[6] R. Tomasello, E. Martinez, R. Zivieri, L. Torres, M. Carpentieri, and G. Finocchio, "A strategy for the design of skyrmion racetrack memories," Scientific Reports 4, 6784 (2014)

[7] O. Tizno, A. R. J. Marshall, N. Fernández-Delgado, M. Herrera, S. I. Molina, and M. Hayne, "Roomtemperature Operation of Low-voltage, Non-volatile, Compound-semiconductor Memory Cells," Scientific Reports 9, 8950 (2019)

[8] A. Ghosh, G. Koster, and G. Rijnders, "Multistability in bistable ferroelectric materials toward adaptive applications," Advanced Functional Materials 26, 5748 (2016).

[9] M. Gu, X. Li, and Y. Cao, "Optical storage arrays: a perspective for future big data storage," Light: Science \& Applications 3, e177 (2014)

[10] J. F. Scott, "Multiferroic memories," Nat. Mater. 6, 256 (2007)

[11] N. A. Spaldin and R. Ramesh, "Advances in magnetoelectric multiferroics," Nat. Mater. 18, 203 (2019)

[12] D. Khomskii, "Classifying multiferroics: Mechanisms and effects," Physics 2, 20 (2009)

[13] M. Fiebig, T. Lottermoser, D. Meier, and M. Trassin, "The evolution of multiferroics," Nature Reviews Materials 1, 16046 (2016)

[14] I. E. Dzyaloshinskii, "A thermodynamic theory of "weak" ferromagnetism of antiferromagnetics," J. Phys. Chem. Solids 4, 241 (1958).
[15] T. Moriya, "Anisotropic Superexchange Interaction and Weak Ferromagnetism," Phys. Rev. 120, 91 (1960).

[16] M. Mostovoy, "Ferroelectricity in Spiral Magnets," Phys. Rev. Lett. 96, 067601 (2006).

[17] J. Stein, M. Baum, S. Holbein, T. Finger, T. Cronert, C. Tölzer, T. Fröhlich, S. Biesenkamp, K. Schmalzl, P. Steffens, C. H. Lee, and M. Braden, "Control of chiral magnetism through electric fields in multiferroic compounds above the long-range multiferroic transition," Phys. Rev. Lett. 119, 177201 (2017)

[18] M. Baum, J. Leist, T. Finger, K. Schmalzl, A. Hiess, L. P. Regnault, P. Becker, L. Bohatý, G. Eckold, and M. Braden, "Kinetics of the multiferroic switching in $\mathrm{MnWO}_{4}, "$ Phys. Rev. B 89, 1 (2014).

[19] T. Hoffmann, P. Thielen, P. Becker, L. Bohatý, and M. Fiebig, "Time-resolved imaging of magnetoelectric switching in multiferroic $\mathrm{MnWO}_{4}$," Phys. Rev. B 84, $184404(2011)$

[20] J. Stein, S. Biesenkamp, T. Cronert, T. Fröhlich, J. Leist, K. Schmalzl, and M. Braden, "Combined arrheniusmerz law describing domain relaxation in type-ii multiferroics," submitted.

[21] D. Niermann, C. P. Grams, M. Schalenbach, P. Becker, L. Bohatý, J. Stein, M. Braden, and J. Hemberger, "Domain dynamics in the multiferroic phase of $\mathrm{MnWO}_{4}$," Phys. Rev. B 89, 134412 (2014).

[22] T. Finger, D. Senff, K. Schmalzl, W. Schmidt, L. P. Regnault, P. Becker, L. Bohatý, and M. Braden, "Electricfield control of the chiral magnetism of multiferroic $\mathrm{MnWO}_{4}$ as seen via polarized neutron diffraction," Phys. Rev. B 81, 1 (2010)

[23] S. Biesenkamp, N. Qureshi, Y. Sidis, P. Becker, L. Bohatý, and M. Braden, "Structural dimerization in the commensurate magnetic phases of $\mathrm{NaFe}\left(\mathrm{WO}_{4}\right)_{2}$ and $\mathrm{MnWO}_{4}, "$ Phys. Rev. B 102, 144429 (2020).

[24] R. Kajimoto, H. Yoshizawa, H. Shintani, T. Kimura, and Y. Tokura, "Magnetic structure of $\mathrm{TbMnO}_{3}$ by neutron diffraction," Phys. Rev. B 70, 012401 (2004).

[25] T. Kimura, G. Lawes, T. Goto, Y. Tokura, and A. P. Ramirez, "Magnetoelectric phase diagrams of orthorhombic $R \mathrm{MnO}_{3}(R=\mathrm{Gd}, \mathrm{Tb}$, and Dy)," Phys. Rev. B 71, 224425 (2005) 
[26] O. Prokhnenko, R. Feyerherm, M. Mostovoy, N. Aliouane, E. Dudzik, A. U. B. Wolter, A. Maljuk, and D. N. Argyriou, "Coupling of Frustrated Ising Spins to the Magnetic Cycloid in Multiferroic $\mathrm{TbMnO}_{3}$," Phys. Rev. Lett. 99, 177206 (2007)

[27] S. Jodlauk, P. Becker, J. A. Mydosh, D. I. Khomskii, T. Lorenz, S. V. Streltsov, D. C. Hezel, and L. Bohatý, "Pyroxenes: a new class of multiferroics," Journal of Physics: Condensed Matter 19, 432201 (2007).

[28] M. Baum, K. Schmalzl, P. Steffens, A. Hiess, L. P. Regnault, M. Meven, P. Becker, L. Bohatý, and M. Braden, "Controlling toroidal moments by crossed electric and magnetic fields," Phys. Rev. B 88, 024414 (2013).

[29] I. Kim, B.-G. Jeon, D. Patil, S. Patil, G. Nénert, and K. H. Kim, "Observation of multiferroic properties in pyroxene $\mathrm{NaFeGe}_{2} \mathrm{O}_{6}$," Journal of Physics: Condensed Matter 24, 306001 (2012)

[30] L. Ding, C. V. Colin, C. Darie, and P. Bordet, "SrMGe $\mathrm{O}_{6}(\mathrm{M}=\mathrm{Mn}$, Co): a family of pyroxene compounds displaying multiferroicity," J. Mater. Chem. C 4, 4236 (2016)

[31] M. Baum, A. C. Komarek, S. Holbein, M. T. FernándezDíaz, G. André, A. Hiess, Y. Sidis, P. Steffens, P. Becker, L. Bohatý, and M. Braden, "Magnetic structure and multiferroic coupling in pyroxene nafesi ${ }_{2} \mathrm{O}_{6}$, Phys. Rev. B 91, 214415 (2015)

[32] H. Katsura, N. Nagaosa, and A. Balatsky, "Spin current and magnetoelectric effect in noncollinear magnets," Phys. Rev. Lett. 95, 57205 (2005)

[33] L. Ding, P. Manuel, D. D. Khalyavin, F. Orlandi, and A. A. Tsirlin, "Unraveling the complex magnetic structure of multiferroic pyroxene $\mathrm{NaFeGe}_{2} \mathrm{O}_{6}$ : A combined experimental and theoretical study," Phys. Rev. B 98, 094416 (2018)

[34] T. V. Drokina, G. A. Petrakovskii, L. Keller, J. Schefer, A. D. Balaev, A. V. Kartashev, and D. A. Ivanov, "Modulated magnetic structure in quasi-one-dimensional clinopyroxene $\mathrm{NaFeGe}_{2} \mathrm{O}_{6}$," Journal of Experimental and Theoretical Physics 112, 121 (2011).

[35] G. J. Redhammer, A. Senyshyn, M. Meven, G. Roth, S. Prinz, A. Pachler, G. Tippelt, C. Pietzonka, W. Treutmann, M. Hoelzel, B. Pedersen, and G. Amthauer, "Nuclear and incommensurate magnetic structure of $\mathrm{NaFeGe}_{2} \mathrm{O}_{6}$ between $5 \mathrm{~K}$ and $298 \mathrm{~K}$ and new data on multiferroic $\mathrm{NaFeSi}_{2} \mathrm{O}_{6}$," Phys. Chem. Miner. 38, 139 (2011)

[36] M. Ackermann, L. Andersen, T. Lorenz, L. Bohatý, and P. Becker, "Anisotropy study of multiferroicity in the pyroxene $\mathrm{NaFeGe}_{2} \mathrm{O}_{6}$," New Journal of Physics 17, 013045 (2015)

37] L. Solovyova and V. Bakakin, "X-ray investigation of Na, Fe-metagermanate $\mathrm{NaFeGe}_{2} \mathrm{O}_{6}$," Krystallographiya 12, 591 (1967).

[38] K. Momma and F. Izumi, "VESTA3 for threedimensional visualization of crystal, volumetric and morphology data," Journal of Applied Crystallography 44, $1272(2011)$

[39] M. Janoschek, S. Klimko, R. Gähler, B. Roessli, and P. Böni, "Spherical neutron polarimetry with mupad,"
Physica B: Condensed Matter 397, 125 (2007)

[40] M. Liu, L. Lin, Y. Zhang, S. Li, Q. Huang, V. O. Garlea, T. Zou, Y. Xie, Y. Wang, C. Lu, L. Yang, Z. Yan, X. Wang, S. Dong, and J.-M. Liu, "Cycloidal magnetism driven ferroelectricity in double tungstate $\mathrm{LiFe}\left(\mathrm{WO}_{4}\right)_{2}$," Phys. Rev. B 95, 195134 (2017)

[41] S. Biesenkamp, D. Gorkov, D. Brüning, A. Bertin, T. Fröhlich, X. Fabrèges, A. Gukasov, M. Meven, P. Becker, L. Bohatý, T. Lorenz, and M. Braden, "Single-crystal investigations on the multiferroic material LiFe $\left(\mathrm{WO}_{4}\right)_{2}$," Phys. Rev. B 103, 134412 (2021)

[42] J. M. Perez-Mato, J. L. Ribeiro, V. Petricek, and M. I. Aroyo, "Magnetic superspace groups and symmetry constraints in incommensurate magnetic phases," Journal of Physics: Condensed Matter 24, 163201 (2012)

[43] I. Urcelay-Olabarria, J. Perez-Mato, J. Ribeiro, J. Garcia-Munoz, E. Ressouche, V. Skumryev, and A. Mukhin, "Incommensurate magnetic structures of multiferroic $\mathrm{MnWO}_{4}$ studied within the superspace formalism," Phys. Rev. B 87, 014419 (2013).

[44] Y. Ishibashi and Y. Takagi, "Note on ferroelectric domain switching," Journal of the Physical Society of Japan 31, 506 (1971)

[45] Y. Ishibashi, "A model of polarization reversal in ferroelectrics," Journal of the Physical Society of Japan 59, 4148 (1990)

[46] M. Avrami, "Kinetics of phase change. i general theory," The Journal of Chemical Physics 7, 1103 (1939).

[47] M. Avrami, "Kinetics of phase change. ii transformationtime relations for random distribution of nuclei," The Journal of Chemical Physics 8, 212 (1940)

[48] M. Avrami, "Granulation, phase change, and microstructure kinetics of phase change. iii," The Journal of Chemical Physics 9, 177 (1941).

[49] F. Kagawa, N. Minami, S. Horiuchi, and Y. Tokura, "Athermal domain-wall creep near a ferroelectric quantum critical point," The Journal of Chemical Physics 7, 10675 (2016)

[50] J. Brooke, T. F. Rosenbaum, and G. Aeppli, "Tunable quantum tunnelling of magnetic domain walls," Nature 413, 610 (2001)

[51] R. C. Miller and A. Savage, "Velocity of Sidewise $180^{\circ}$ Domain-Wall Motion in $\mathrm{BaTiO}_{3}$ as a Function of the Applied Electric Field," Phys. Rev. 112, 755 (1958).

[52] J. F. Scott, Ferroelectric Memories, Advanced microelectronics (Springer, 2000).

[53] D. Meier, N. Leo, M. Maringer, T. Lottermoser, and M. Fiebig, "Topology and manipulation of multiferroic hybrid domains in $\mathrm{MnWO}_{4}$," Phys. Rev. B 80, 1 (2009).

[54] M. Matsubara, S. Manz, M. Mochizuki, T. Kubacka, A. Iyama, N. Aliouane, T. Kimura, S. L. Johnson, D. Meier, and M. Fiebig, "Magnetoelectric domain control in multiferroic $\mathrm{TbMnO}_{3}$," Science 348, 1112 (2015).

[55] Data are available at https://doi.ill.fr/10.5291/ ILL-DATA.CRG-2439 